\title{
Numerical Investigation of Nanostructured Silica PCFs for Sensing Applications
}

\author{
JINCY JOHNY, ${ }^{1}$ RADHAKRISHNA PRABHU $10,{ }^{1,2}$ and WAI KEUNG FUNG ${ }^{1}$ \\ 1.-School of Engineering, Robert Gordon University, Aberdeen, UK. 2.—e-mail: r.prabhu@rgu.ac.uk
}

Photonic crystal fibers (PCFs) developed using nanostructured composite materials provide special optical properties. PCF light propagation and modal characteristics can be tailored by modifying their structural and material parameters. Structuring and infusion of liquid crystal materials enhances the capabilities of all silica PCFs, facilitating their operation in different spectral regimes. The wavelength tunability feature of nanostructured PCFs can be utilized for many advanced sensing applications. This paper discusses a new approach to modify the optical properties of PCFs by periodic nanostructuring and composite material (liquid crystal-silica) infiltration. PCF characteristics like confinement wavelength, confinement loss, mode field diameter (MFD) and bandwidth are investigated by varying the structural parameters and material infiltrations. Theoretical study revealed that composite material infusion resulted in a spectral band shift accompanied by an improvement in PCF bandwidth. Moreover, nanostructured PCFs also achieved reduced confinement losses and improved MFD which is very important in long-distance remote sensing applications.

\section{INTRODUCTION}

The demand for optical fibers and fiber optic technologies increased considerably after the telecommunication revolution which ignited in the 1980's. The exceptional characteristics of optical fibers such as structural versatility and enhanced sensitivity over current techniques make them a suitable candidate for sensing applications. They have many advantages such as immunity from electromagnetic interference, electrical isolation, small size, remote sensing ability, multiplexing capability, freedom from corrosion and ability to operate in extreme environmental conditions. ${ }^{1}$ Despite the fact that conventional optical fibers offer remarkable performance in the communication industry, they do not satisfy all the needs of sensing industry. To overcome these limitations of communication fibers, speciality fibers like photonic crystal fibers (PCF), doped fibers, etc. are emerging for advanced sensing applications.

$\mathrm{PCFs}^{2}$ have been gaining in popularity in recent years, owing to their specialized geometrical structure (core-air hole cladding) and unique properties, which include their guiding mechanisms and modal characteristics. $^{3}$ They produce lower optical transmission losses compared to standard silica optical fibers. ${ }^{4}$ PCFs are more flexible than normal optical fibers, because it is possible to manage their properties, leading to a freedom of design. ${ }^{5}$ The large refractive index difference between silica and air of the PCF enables tighter mode confinements than conventional all-solid single-mode glass optical fibers. ${ }^{6}$ Commercially available PCFs, fabricated from silica $\left(\mathrm{SiO}_{2}\right)$ material, operate mostly in the communication wavelength band $(1.55 \mu \mathrm{m})$, limiting their scope for advanced fiber-optic sensing applications which aims the near-IR (NIR) and midIR (MIR) wavelengths. Material absorption limits of silica have always been a major problem in the large wavelength region. ${ }^{7}$ All these drawbacks of $\mathrm{SiO}_{2}$ material create a need for new materials for emerging fiber optic applications like NIR and MIR (3$6 \mu \mathrm{m})$ sensors, next generation communication systems in the $2.5-\mu \mathrm{m}$ wavelength band, MIR fiber lasers, etc. ${ }^{8,9}$ Many novel optical glasses like chalcogenide, tellurite and fluoride glasses offer enhanced performances, finding numerous applications in MIR wavelengths. ${ }^{10,11}$ 
The properties of $\mathrm{SiO}_{2}$-based PCF can be manipulated by two techniques-nanostructuring and fusing in liquid crystal-silica composite materials. The modified $\mathrm{SiO}_{2}$ material shows some fantastic optical properties wherein we can tune the spectral bands to the required wavelength region. Wavelength-scale periodic microstructuring creates a dramatic change in the optical properties of the materials. ${ }^{6}$ Nanostructured optical materials will provide special properties which can improve the capabilities of existing optical sensing technologies. ${ }^{12,13}$ Nanostructuring of PCF air holes is expected to improve their birefringence property, reduce the confinement losses and also decrease their effective mode area, which in turn improves the propagation distance and range of the fiber sensor. ${ }^{14}$ Highly birefringent PCFs exhibit multiparameter sensing capabilities as they have different polarization states which show different sensitivities to physical parameters like temperature and strain. ${ }^{15}$ Furthermore, addition of liquid crystal materials into the cladding holes modifies PCF transmission and polarization properties, enabling them to operate within the photonic bands having the highest sensitivities. Another advantage of material infusion is that it is possible to modify the properties of PCFs even after fabrication. PCF air holes filled with special materials like liquid crystals change their optical properties in response to electric or magnetic fields or light intensity. Liquid crystal materials infused into the cladding holes creates a PBG (photonic bandgap) effect, restricting the modes within the core region rather than leaking, facilitating a stronger sensing signal. ${ }^{16}$

NIR wavelengths of the optical spectrum are of particular interest for fiber-optic sensing applications due to their improved sensitivity and accuracy compared to other spectral regions. The NIR bands have shown high sensitivities to methane gas sensing, which is of great interest in many industrial and safety applications. ${ }^{17,18}$ Gas detection is a very crucial task in the oil and gas industry, in order to protect their valuable assets and workers from safety hazards. The MIR wavelengths have attracted different laser spectroscopic-based environmental and medical sensing applications. ${ }^{19,20}$ Considering the growing interest of optical fibers in new application areas, it becomes important to enhance their capabilities and functionalities. Future applications will rely on well-developed optical materials and technologies along with new, modified and special properties of optical fibers. ${ }^{8}$

This paper investigates the effects of geometrical structure and infused materials to tune critical parameters of silica PCFs like operating wavelength, confinement loss, MFD and bandwidth. These parameters are very important in fiber-optic sensing applications as they affect the sensitivity, range and spatial coverage of the sensor. Through this study, we were able to achieve a shifting and broadening of the PCF spectral bands by nanostructuring and infusing composite materials within the PCF.

\section{THEORY}

PCFs normally have two modes of operation based on their light guiding technique: index guiding and bandgap guiding. ${ }^{21}$ Index guiding PCFs operate similarly to conventional optical fibers wherein light is confined within the high index core by a modified total internal reflection principle. ${ }^{22}$ However, bandgap PCFs guide light in the low index core region by the reflection from the photonic crystal cladding. ${ }^{23}$ Confinement loss and effective area are two important propagation characteristics of the PCFs which can be tuned by altering their physical parameters like core diameter $(\rho)$, cladding hole diameter $(d)$ and pitch $(\Lambda)$ in combination with the choice of material refractive index and type of crystal lattice. ${ }^{5,24}$

\section{Confinement Loss}

Confinement or leakage loss $(L)$ is the light confinement ability of the PCF within its core. ${ }^{25}$

$$
L=\frac{\left(\frac{20}{\ln (10)}(2 \pi)\right) \operatorname{Im}\left(\mathrm{n}_{\mathrm{eff}}\right)}{\lambda},
$$

where $\operatorname{Im}\left(n_{\text {eff }}\right)$ is the imaginary part of the effective refractive index, $n_{\mathrm{eff}}$, and $\lambda$ is the propagating wavelength. ${ }^{26}$

\section{Effective Mode Area}

Effective mode area ${ }^{27}$ of the PCF is given by:

$$
A_{\text {eff }}=\frac{\left[\iint_{-\infty}^{\infty}|E(x, y)|^{2} \mathrm{~d} x \mathrm{~d} y\right]^{2}}{\iint_{-\infty}^{\infty}|E(x, y)|^{4} \mathrm{~d} x \mathrm{~d} y},
$$

where $E(x, y)$ is the optical mode field distribution.

Also, effective area is related to MFD by the equation: ${ }^{28}$

$$
A_{\mathrm{eff}}=k_{n}\left(\frac{\pi}{4}\right) \mathrm{MFD}^{2}=k_{n} \pi w^{2},
$$

where $k_{n}$ is the correction factor, and spot size $w=\mathrm{MFD} / 2$.

MFD is approximated as,

$$
\operatorname{MFD} \approx \frac{2}{\sqrt{\pi}} \sqrt{A_{\text {eff }}}
$$

\section{NANOSTRUCTURED PCF MODELING}

Current studies are carried out by changing the size (nano-sized holes in the core and micro-sized holes in the cladding), shape (circular, elliptical) and distribution of PCF air holes. In the previous study, the designed solid core PCFs exhibited 


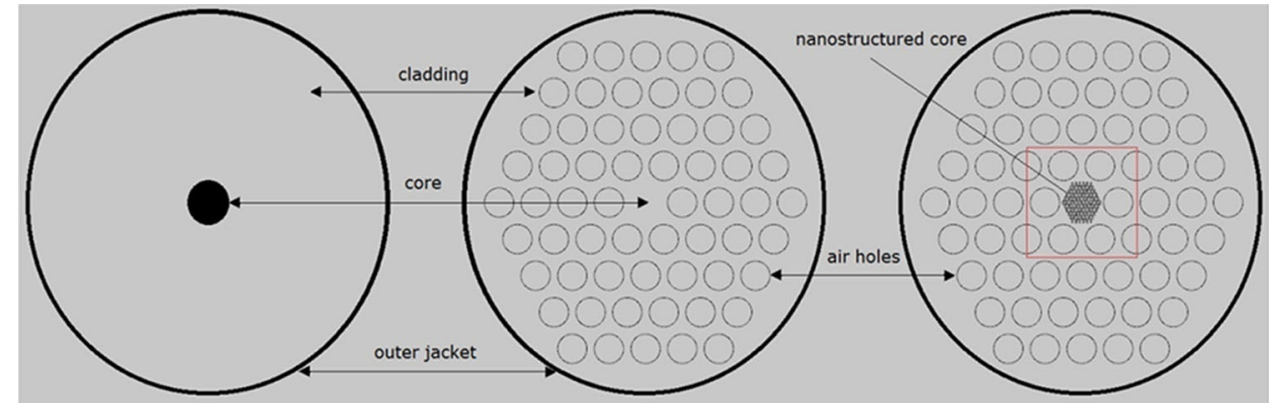

(a)

(b)

(c)

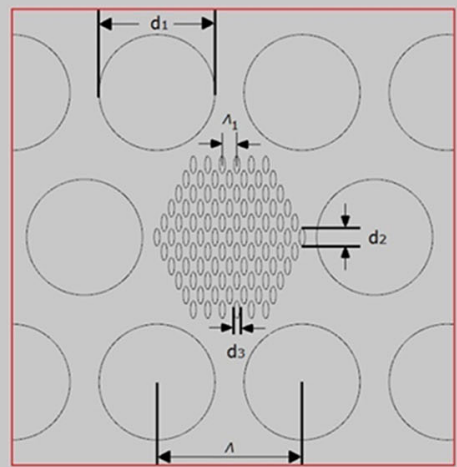

(d)

Fig. 1. Cross-section of: (a) step-index SMF, (b) solid core PCF, (c) designed nanostructured PCF and (d) nanostructured core zoomed view.

limited operating wavelengths. ${ }^{29}$ Hence, in order to achieve spectral shifting and bandwidth enhancement, core nanostructuring and also the effect of different liquid crystal materials infiltrations within the cladding holes are being investigated. PCF parameters such as confinement losses, effective area and MFD are studied as a function of normalized frequency $(\Lambda / \lambda)$. MFD and effective area is associated with the electric field distribution within the fibers. Analysis of these parameters gives a better insight of the propagation characteristics of PCFs.

Figure 1a and $\mathrm{b}$ shows the cross-sectional view of the structural difference between a standard stepindex single-mode fiber (SMF) and a solid core PCF with microstructured cladding. During the fiber modeling, the only physical parameter that needs to be taken into account in the case of SMF is the core diameter. On the other hand, while modeling a PCF, three geometrical parameters are to be considered: core diameter, cladding hole diameter and the pitch (hole-to-hole distance). Figure $1 \mathrm{c}$ and d shows the cross-section of the designed nanostructured PCF with a four-ring hexagonal lattice of circular air holes in the cladding and a five-ring array of elliptical air holes in the core. Simulations were conducted on a hexagonal PCF designed using the wave optics module of COMSOL MULTIPHYSICS 5.1. The geometrical parameters of the nanostructured PCF comprise: pitch $(\Lambda)$ which is the cladding hole center-to-center distance; $d_{1}$, the diameter of the cladding air hole; $d_{2}$ and $d_{3}$, the length of the major and minor axis, respectively, of the elliptical air holes within the core; and $\Lambda^{\prime}$ the hole-to-hole spacing between the elliptical air holes of the core. The elliptical air holes introduce birefringence properties, which greatly improves the sensing capabilities of the PCF.

\section{SIMULATION RESULTS AND DISCUSSION}

Figure 2a and $b$ shows the electric field profile of the fundamental mode obtained for the designed nanostructured PCF. The central core, colored red, indicates the region of the highest electric field. From Table I, we can see that the mode confinement wavelengths can be brought down from the infrared (IR) to the near infrared (NIR) wavelengths by tuning the physical parameters of the core and the cladding air holes. A NIR lower cut-off wavelength of $925 \mathrm{~nm}$ was achieved for the nanostructured PCF with the design parameters: $\Lambda=1 \mu \mathrm{m}, d_{1}=0.8 \mu \mathrm{m}$, $\Lambda^{\prime}=100 \mathrm{~nm}, d_{2}=60 \mathrm{~nm}, d_{3}=20 \mathrm{~nm}$.

Figure $3 \mathrm{a}$ depicts the variation in confinement loss (in $\mathrm{dB} / \mathrm{Km}$ ) with respect to normalized frequency $(v=\Lambda / \lambda)$ for different pitch or cladding hole spacing. It can be observed that confinement losses decrease with normalized frequency, or in other words confinement losses increase with wavelength. Another advantage of bringing down the mode confinement wavelengths from the IR to NIR wavelengths is a reduction in confinement losses. When the confinement loss is reduced, light becomes more confined in the PCF core. Hence, the designed low 


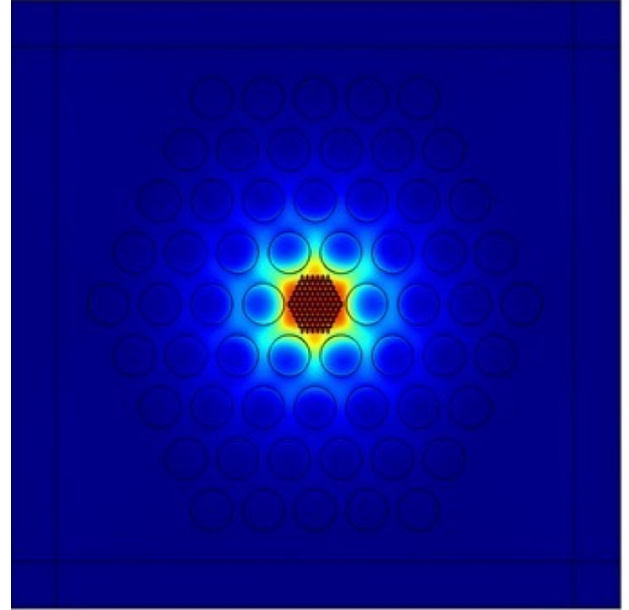

(a)

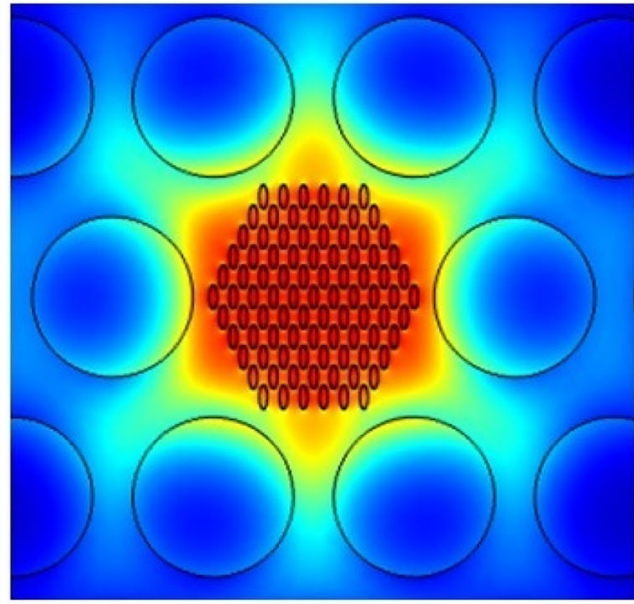

(b)

Fig. 2. (a) Electric field mode profile of the nanostructured PCF, (b) zoomed view of the electric field mode profile in the PCF core

Table I. Confinement wavelengths with varying physical parameters

\begin{tabular}{|c|c|c|c|}
\hline Sl. no. & Core dimensions & Cladding dimensions & Confinement wavelengths $(\mathrm{nm})$ \\
\hline 1 & $\begin{array}{c}\Lambda^{\prime}=0.1 \Lambda=0.22 \mu \mathrm{m}=220 \mathrm{~nm} \\
d_{2}=0.6 \Lambda^{\prime}=0.132 \mu \mathrm{m}=132 \mathrm{~nm} \\
d_{3}=0.2 \Lambda^{\prime}=0.044 \mu \mathrm{m}=44 \mathrm{~nm}\end{array}$ & $\begin{array}{c}\Lambda=2.2 \mu \mathrm{m} \\
d_{1}=0.8 \Lambda=1.76 \mu \mathrm{m}\end{array}$ & $\geq 1950$ \\
\hline 2 & $\begin{array}{c}\Lambda^{\prime}=0.1 \Lambda=0.2 \mu \mathrm{m}=200 \mathrm{~nm} \\
d_{2}=0.6 \Lambda^{\prime}=0.12 \mu \mathrm{m}=120 \mathrm{~nm} \\
d_{3}=0.2 \Lambda^{\prime}=0.04 \mu \mathrm{m}=40 \mathrm{~nm}\end{array}$ & $\begin{array}{c}\Lambda=2 \mu \mathrm{m} \\
d_{1}=0.8 \Lambda=1.6 \mu \mathrm{m}\end{array}$ & $\geq 1775$ \\
\hline 3 & $\begin{array}{l}\Lambda^{\prime}=0.1 \Lambda=0.15 \mu \mathrm{m}=150 \mathrm{~nm} \\
d_{2}=0.6 \Lambda^{\prime}=0.09 \mu \mathrm{m}=90 \mathrm{~nm} \\
d_{3}=0.2 \Lambda^{\prime}=0.03 \mu \mathrm{m}=30 \mathrm{~nm}\end{array}$ & $\begin{array}{c}\Lambda=1.5 \mu \mathrm{m} \\
d_{1}=0.8 \Lambda=1.2 \mu \mathrm{m}\end{array}$ & $\geq 1450$ \\
\hline 4 & $\begin{array}{l}\Lambda^{\prime}=0.1 \Lambda=0.1 \mu \mathrm{m}=100 \mathrm{~nm} \\
d_{2}=0.6 \Lambda^{\prime}=0.06 \mu \mathrm{m}=60 \mathrm{~nm} \\
d_{3}=0.2 \Lambda^{\prime}=0.02 \mu \mathrm{m}=20 \mathrm{~nm}\end{array}$ & $\begin{array}{c}\Lambda=1 \mu \mathrm{m} \\
d_{1}=0.8 \Lambda=0.8 \mu \mathrm{m}\end{array}$ & $\geq 925$ \\
\hline
\end{tabular}

confinement loss PCF returns stronger sensing signals when used in any sensor configuration. Figure $3 \mathrm{~b}$ shows the changes in MFD (in $\mu \mathrm{m}$ ) with respect to normalized frequency for different pitch values. From the graph, it is clear that MFD decreases with normalized frequency, or MFD experiences an increase for longer wavelengths and a decrease for shorter wavelengths. This is because, at lower wavelengths, the power density of the electric field propagating through the fiber would be higher and hence the effective mode area and MFD would be smaller. ${ }^{30}$

Infiltrating different liquid crystal materials like K21 (refractive index, $n_{\mathrm{LC}}=1.732$ ), $\mathrm{PCH}-5$ $\left(n_{\mathrm{LC}}=1.6049\right)$ and Cat No. $1550\left(n_{\mathrm{LC}}=1.522\right)$ into the cladding holes resulted in the shifting and widening of the PCF spectral bands. The confinement wavelengths achieved are: K21 (1650$2000 \mathrm{~nm})$; PCH-5 (1200-1650 nm) and Cat No.
1550 (825-1100 nm). Hence, mode confinements in NIR wavelengths were achieved by infiltrating the holes with liquid crystal material Cat No. 1550. To sum up, PCF nanostructuring and liquid crystal material infiltrations reduce its losses and enhance the PCF sensor transmission distance and coverage.

From Fig. 3c, we can observe the changes in spectral bandwidth corresponding to different liquid crystal refractive indices values. It was observed that altering refractive index of the liquid crystal material filled into the cladding holes of the PCF resulted in an expansion and movement of its photonic bandgap. This feature can be utilised in designing PCFs of any required wavelengths specific to any particular application. Moreover, liquid crystal PCFs owing to their PBG guiding mechanism enable stronger light confinements facilitating sensing at longer distances. 

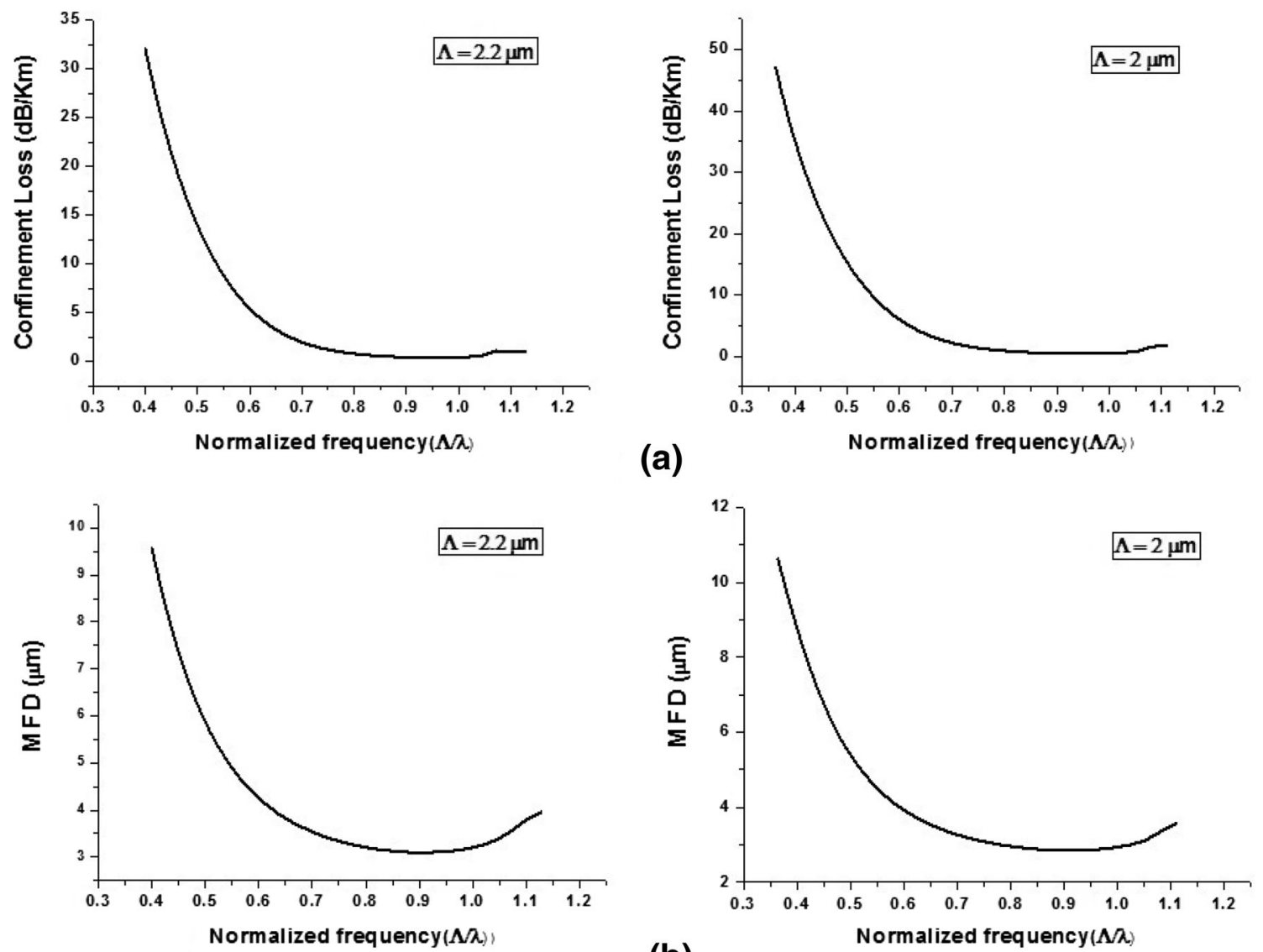

(b)

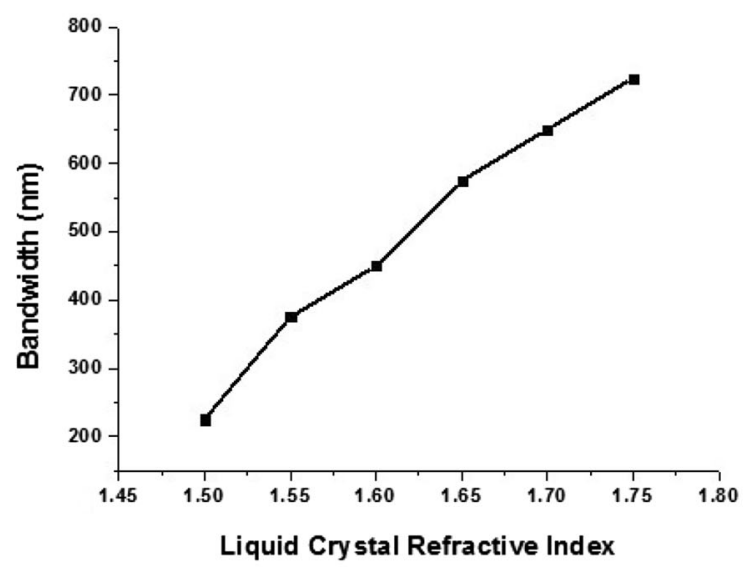

(c)

Fig. 3. (a) Confinement loss versus normalized frequency for $\Lambda=2.2 \mu \mathrm{m}$ and $\Lambda=2 \mu \mathrm{m}$; (b) MFD versus normalized frequency for $\Lambda=2.2 \mu \mathrm{m}$ and $\Lambda=2 \mu \mathrm{m}$; (c) bandwidth versus liquid crystal refractive index.

\section{CONCLUSION}

The computational study carried out by nanostructuring the PCF core and by changing the size, shape and distribution of the PCF holes resulted in a shift in the spectral band, accompanied by a reduction in confinement losses and MFD. It was found that the PCF properties are a strong function of its structural parameters. Further simulations carried out by infusing different liquid crystal composite materials into the PCF resulted in a wavelength shift from IR to NIR. Moreover, altering the refractive index of the liquid crystal material filled into the cladding holes of the PCF caused a shifting and broadening of the photonic bands. Through these simulations, it was identified that the spectral positions and bandgaps can be tuned by 
nanostructuring the PCF holes and changing the material infiltrations. Hence, nanostructuring and composite material infiltrations make it possible to have different designer wavelengths for the PCF sensors. In addition, low confinement losses and MFD improve the signal power of the sensor, which in turn enhances its range and propagation distance. This study can be extended to MIR wavelengths giving scope for future fiber-optic sensing and next-generation communication applications.

\section{OPEN ACCESS}

This article is distributed under the terms of the Creative Commons Attribution 4.0 International License (http://creativecommons.org/licenses/by/4.0/), which permits unrestricted use, distribution, and reproduction in any medium, provided you give appropriate credit to the original author(s) and the source, provide a link to the Creative Commons license, and indicate if changes were made.

\section{REFERENCES}

1. B. Lee, Opt. Fiber Technol. 9, 57 (2003).

2. J.C. Knight, T.A. Birks, P.S.J. Russell, and D.M. Atkin, Opt. Lett. 21, 1547 (1996).

3. R. Buczynski, Acta Phys. Pol., A 106, 141 (2004).

4. J.C. Knight, Nature 424, 847 (2003).

5. A.M.R. Pinto and M. Lopez-Amo, J. Sens. 2012, 598178 (2012). doi:10.1155/2012/598178.

6. P. Dainese, P.S.J. Russell, N. Joly, J.C. Knight, G.S Wiederhecker, H.L. Fragnito, V. Laude, and A. Khelif, Nat. Phys. 2, 388 (2006).

7. J.D. Shephard, A. Urich, R.M. Carter, P. Jaworski, R.R. Maier, W. Belardi, F. Yu, W.J. Wadsworth, J.C. Knight, and D.P. Hand, Front. Phys. 3, 24 (2015).

8. K. Schuster, S. Unger, C. Aichele, F. Lindner, S. Grimm, D. Litzkendorf, J. Kobelke, J. Bierlich, K. Wondraczek, and H. Bartelt, Adv. Opt. Technol. 3, 447 (2014).
9. F.K. Tittel, D. Richter, and A. Fried, Solid-state Mid-infrared Laser Sources, ed. I.T. Sorokina and K.L. Vodopyanov (Berlin Heidelberg: Springer, 2003), pp. 458-529.

10. B. Mizaikoff, Anal. Chem. 75A, 258 (2003).

11. J. Ballato and P. Dragic, J. Am. Ceram. Soc. 96, 2675 (2013).

12. Y. Zhang, Physical Properties Investigation of Nanostructured Materials and Their Applications (Ann Arbor: ProQuest, 2008), pp. 1-2.

13. C. Paquet and E. Kumacheva, Mater. Today 11, 48 (2008).

14. D. Chen, in International Symposium on Biophotonics, Nanophotonics and Metamaterials, 362-365 (2006).

15. T. Nasilowski, P. Lesiak, R. Kotynski, M. Antkowiak, A.F. Fernandez, F. Berghmans and H. Thienpont: Proceedings of Laser and Electro-Optics Society, 29-32 (2003).

16. T.R. Wolinski, K. Szaniawska, S. Ertman, P. Lesiak, A.W. Domanski, R. Dabrowski, E. Nowinowski-Kruszelnicki, and J. Wojcik, Meas. Sci. Technol. 17, 985 (2006).

17. A.M. Cubillas, J.M. Lazaro, O.M. Conde, M.N. Petrovich, and J.M. Lopez-Higuera, Sensors 9, 6261 (2009).

18. J. Shemshad, S.M. Aminossadati, and M.S. Kizil, Sens. Actuators, B 171, 77 (2012).

19. M.W. Sigrist, J. Adv. Res. 6, 529 (2015).

20. B. Jean and T. Bende, Solid-State Mid-Infrared Laser Sources, ed. I.T. Sorokina and K.L. Vodopyanov (Berlin Heidelberg: Springer, 2003), pp. 530-565.

21. B.P. Pal, Guided Wave Optical Components and Devices: Basics, Technology, and Applications, 2nd ed. (San Diego: Academic press, 2010), pp. 51-64.

22. R.B. Wehrspohn, H. Kitzerow, and K. Busch, Nanophotonic Materials: Photonic Crystals, Plasmonics, and Metamaterials (New Jersey: Wiley, 2008), pp. 291-293.

23. L. Xiao, W. Jin, and M.S. Demokan, Opt. Express 15, 15637 (2007).

24. K. Ahmed and M. Morshed, Sens. Biosens. Res. 7, 1 (2016).

25. S. Olyaee, M. Seifouri, A. Nikoosohbat, and M.S.E. Abadi, Int. J. Chem. Nucl. Mater. Metall. Eng. 9, 253 (2015).

26. X.L. Tan, Y.F. Geng, Z. Tian, P. Wang, and J.Q. Yao, $O p$ toelectron. Lett. 5, 124 (2009).

27. K. Miyagi, Y. Namihira, S.A. Razzak, S.F. Kaijage, and F. Begum, Opt. Rev. 17, 388 (2010).

28. N.A. Mortensen, Opt. Express 10, 341 (2002).

29. J. Johny, R. Prabhu, and W.K. Fung, Opt. Quantum Electron. 48, 1 (2016).

30. R. Billington, Effective Area of Optical Fibres-Definition and Measurement Techniques (Teddington, Middlesex: Centre for Optical and Environmental Metrology, National Physical Laboratory, 1999). 